\title{
Factors Underlying Quality Problems with Alcohol Screening Prompted by a Clinical Reminder in Primary Care: A Multi-site Qualitative Study
}

\author{
Emily C. Williams, PhD, MPH ${ }^{1,5}$, Carol E. Achtmeyer, MN, ARNP ${ }^{1,2,3}$, Rachel M. Thomas, $\mathrm{MPH}^{7}$, \\ Joel R. Grossbard, $P h D^{3}$, Gwen T. Lapham, PhD, MSW, MPH', , Laura J. Chavez, MPH', \\ Evette J. Ludman, $\mathrm{PhD}^{6,7}$, Douglas Berger, MD, M Litt ${ }^{2,4}$, and Katharine A. Bradley, MD, MPH ${ }^{1,3,4,5,7}$
}

\begin{abstract}
'Health Services Research \& Development (HSR\&D), Center of Innovation for Veteran-Centered and Value-Driven Care, Veterans Affairs (VA) Puget Sound Health Care System, Seattle, WA, USA; ${ }^{2}$ Primary and Specialty Medical Care Services, Veterans Affairs (VA) Puget Sound Health Care System, Seattle, WA, USA; ${ }^{3}$ Center of Excellence in Substance Abuse Treatment and Education (CESATE), Veterans Affairs (VA) Puget Sound Health Care System, Seattle, WA, USA; ${ }^{4}$ Department of Medicine, University of Washington, Seattle, WA, USA; ${ }^{5}$ Department of Health Services, University of Washington, Seattle, WA, USA; ${ }^{6}$ Department of Psychiatry and Behavioral Sciences, University of Washington, Seattle, WA, USA; 7 Group Health Research Institute, Seattle, WA, USA.
\end{abstract}

BACKGROUND: Population-based alcohol screening is recommended in primary care, and increasingly incentivized by policies, yet is challenging to implement. The U.S. Veterans Health Administration (VA) achieved high rates of screening using a national performance measure and associated electronic clinical reminder to prompt and facilitate screening and document results. However, the sensitivity of alcohol screening for identifying unhealthy alcohol use is low in VA clinics.

OBJECTIVE: We aimed to understand factors that might contribute to low sensitivity of alcohol screening.

DESIGN: This was an observational, qualitative study.

PARTICIPANTS: Participants included clinical staff responsible for conducting alcohol screening and nine independently managed primary care clinics of a single VA medical center in the Northwestern U.S.

APPROACH: Four researchers observed clinical staff as they conducted alcohol screening. Observers took handwritten notes, which were transcribed and coded iteratively. Template analysis identified a priori and emergent themes.

KEY RESULTS: We observed 72 instances of alcohol screening conducted by 31 participating staff. Observations confirmed known challenges to implementation of care using clinical reminders, including workflow and flexibility limitations. Three themes specific to alcohol screening emerged. First, most observed screening was conducted verbally, guided by the clinical reminder, although some variability in approaches to screening (e.g., paperbased or laminate-based screening) was observed. Second, specific verbal screening practices that might contribute to low sensitivity of clinical screening were identified, including conducting non-verbatim screening and making inferences, assumptions, and/or suggestions to input

Views expressed in this article are those of the authors and do not necessarily represent the views of the Department of Veterans Affairs or of the University of Washington.

Received August 19, 2014

Revised January 28, 2015

Accepted February 5, 2015

Published online March 3, 2015 responses. Third, staff introduced and adapted screening questions to enhance patient comfort.

CONCLUSIONS: This qualitative study in nine clinics found that implementation of alcohol screening facilitated by a clinical reminder resulted primarily in verbal screening in which questions were not asked vertbatim and were otherwise adapted. Non-verbal approaches to screening, or patient self-administration, may enhance validity and standardization of screening while simultaneously addressing limitations of the clinical reminder and issues related to perceived discomfort.

KEY WORDS: alcohol screening; qualitative; clinical reminders; quality. J Gen Intern Med 30(8):1125-32 DOI: $10.1007 / \mathrm{s} 11606-015-3248-\mathrm{Z}$

๑) Society of General Internal Medicine 2015

\section{INTRODUCTION}

Routine population-based alcohol screening is recommended in primary care settings in order to identify patients with unhealthy alcohol use who may benefit from brief alcohol intervention. ${ }^{1-3}$ While together screening and brief intervention are considered a top prevention priority for U.S. adults, ${ }^{4,5}$ they have proven challenging to implement in routine care. ${ }^{5-8}$

The U.S. Veterans Health Administration (VA) has achieved high rates of documented alcohol screening, both overall $^{9,10}$ and relative to other healthcare systems. ${ }^{6}$ As a result, the VA has been highlighted as a leader in implementation of alcohol screening. ${ }^{11}$ Consistent with implementation of other clinical services, ${ }^{12,13}$ the VA used a combination of a national performance measure and an electronic clinical reminder to implement alcohol screening. Specifically, the VA implemented a national performance measure incentivizing annual screening with the validated Alcohol Use Disorders Identification Test - Consumption (AUDIT-C) questionnaire ${ }^{14-18}$ and disseminated an associated clinical reminder (Fig. 1), to be embedded in the electronic medical record. ${ }^{13}$ While there is no "gold standard" for optimal use of the 


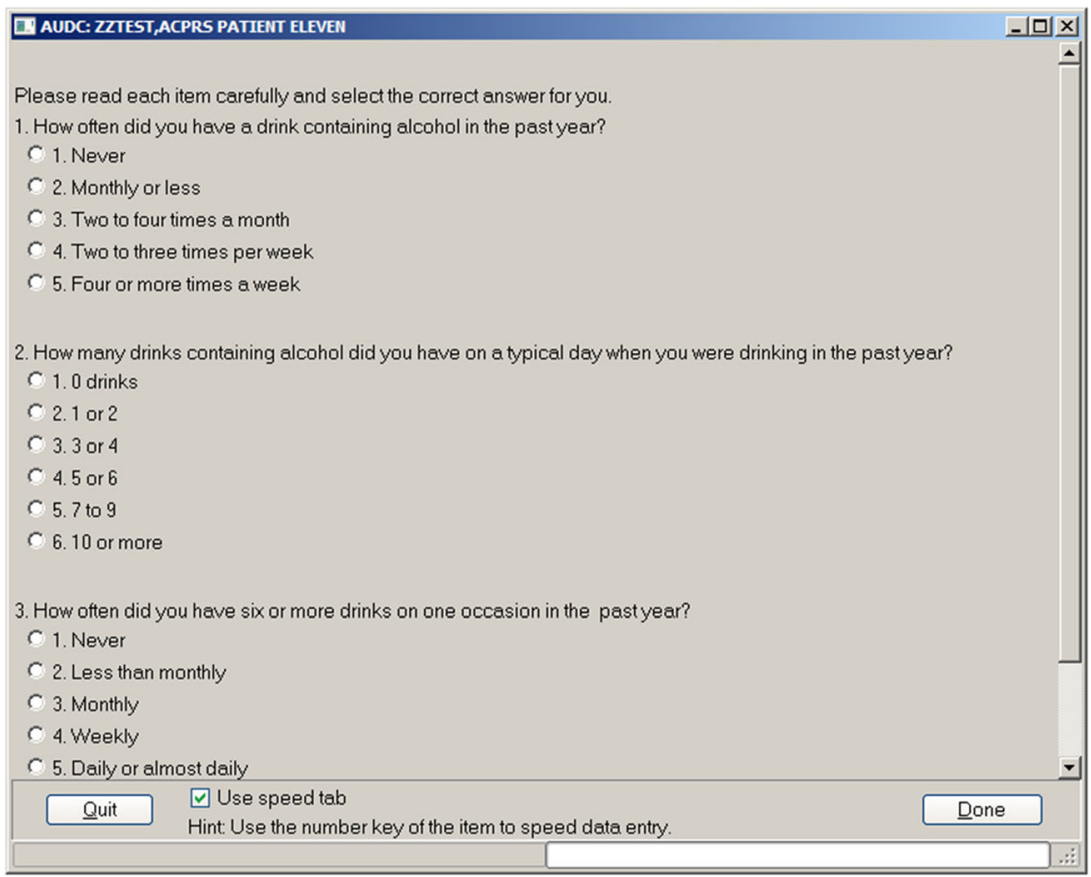

Figure 1. Screen capture of the U.S. Veteran Health Administration's (VA's) electronic clinical reminder for alcohol screening with the Alcohol Use Disorders Identification Test - Consumption (AUDIT-C) questionnaire.

AUDIT-C clinical reminder, it was designed to: 1) prompt annual clinical screening; 2) guide clinical staff to perform screening in a validated, standardized, reproducible way; 3) automatically score and document results of screening; and 4) when positive, trigger a subsequent clinical reminder for brief intervention and other appropriate follow-up. The AUDIT-C clinical reminder was used 1.5 million times in its first year (2004), and over 24 million screens have been documented with the clinical reminder in the last five years. ${ }^{9,10}$

However, while the AUDIT-C has high sensitivity for identifying unhealthy alcohol use based on validation studies, ${ }^{14,16,18}$ the sensitivity of alcohol screening performed in VA clinics appears to be lower than expected. ${ }^{19,20}$ Specifically, a previous study found that $61 \%$ of patients who screened positive for unhealthy alcohol use on confidential mailed surveys screened negative when screened clinically. ${ }^{20}$ However, little is known about how screening is conducted in practice and what resulted in this discrepancy.

New policy initiatives in the U.S. are paving the way for widespread implementation of alcohol screening. The Centers for Medicare \& Medicaid Services (CMS) now reimburse for annual alcohol screening, ${ }^{21}$ and the Affordable Care Act (ACA) established both alcohol screening and brief intervention as standard preventive benefits. ${ }^{21-23}$ Other healthcare systems are beginning to use strategies similar to those used by the VA to implement alcohol screening, ${ }^{24}$ and more are likely to do so in response to these policy initiatives. Understanding factors underlying the low sensitivity of clinical alcohol screening in VA may help optimize the quality of screening being implemented in VA and other systems. The objective of this study was to observe clinical staff as they conducted alcohol screening in order to understand factors that might contribute to low sensitivity of clinical screening.

\section{METHODS}

\section{Setting}

This observational qualitative study was conducted at nine primary care clinics located at seven geographically distinct sites within a single VA Healthcare System in the Northwestern United States. This included two large medical centers, each with a large primary care clinic and a women's clinic that provides primary care, two VA-managed community-based outpatient clinics (CBOCs), and three contract CBOCs (i.e., local clinics that offer VA care to Veterans). Similar to other VA primary care settings, clinical staff, including Registered Nurses (RNs), Licensed Practicing Nurses (LPNs), and Health Technicians (Health Techs), are responsible for conducting alcohol screening during patient intake at these sites. The AUDIT-C electronic clinical reminder is "due" annually and "triggered" 9 months after the previous screen. ${ }^{25}$ No preferred approach to screening (e.g., paper questionnaire or in-person verbal interview) has been specified by national VA policy, but it is common for care incentivized by performance measures to be documented using electronic clinical reminders. ${ }^{25}$

\section{Participants}

Clinical staff responsible for alcohol screening were recruited opportunistically at each site and asked for permission to be observed during their usual course of clinical care. Either 
before or on the first day of observation at each clinic, clinical staff were given an overview of the study, and those who considered participating were given a one-page information statement describing the study. Staff participants verbally consented to be observed; written consent was not obtained to ensure that no identifying information regarding participants was collected. Patients were not considered participants of the study and were not recruited. However, because patients were observed in the course of observing clinical staff who participated in the study, enrolled staff participants asked their patients whether they were comfortable being observed, and encounters in which the patient also agreed to the observation were observed. Clinical staff participants were told that observers were interested in understanding the ways in which clinicians interact with clinical reminders, but were blinded to the study's specific focus on alcohol-related care. The study, including a waiver of written informed consent, was reviewed and approved by the Institutional Review Board at VA Puget Sound.

\section{Observational Data Collection}

Between July 2010 and January 2011, four masters' level researchers trained in public health (RT, GL, LC), social work (GL), and/or nursing (CA), observed clinical staff as they interacted with clinical reminders during intake in order to specifically observe alcohol screening. Observers were not blinded to the study purpose. Based on methods previously used in VA, ${ }^{26,27}$ observers spent 1-2 days at each of the nine clinics and took handwritten ethnographic notes of their observations. Notes were observational, not interpretive, and included short descriptions of what the observer saw that was pertinent to the use of clinical reminders and/ or alcohol screening. Though not solicited, participating clinical staff occasionally offered their opinions regarding clinical reminders and/or alcohol screening, and these were also documented in observers' notes. Observations were completed until saturation (no new information was being obtained).

\section{Qualitative Analyses}

Handwritten observational notes were transcribed into Microsoft Word ${ }^{\circledR}$ by each individual observer. Data were analyzed in an iterative fashion using template analysis (also called codebook analysis or thematic coding). ${ }^{28}$ While some codes are identified a priori, others emerge from the data. In this way, template analysis is midway between content analysis, where all codes are rigidly defined a priori, and analytic approaches based on grounded theory, where all codes emerge from the data. ${ }^{29}$ The coding template was initially based on both Greenhalgh et al.'s conceptual model of diffusion of innovations in services settings, ${ }^{30}$ and a summary of previous literature describing barriers to and facilitators of use of clinical reminders for implementation of evidence-based care. ${ }^{9,10,26,27,31-37}$ Greenhalgh's model identified broad domains of implementation (e.g., the implementation process), while previous literature regarding the use of clinical reminders for implementation identified specific factors (e.g., workflow) that might contribute to low sensitivity of clinical screening within those broad domains. The template was iteratively revised based on initial and ongoing review of field notes by all investigators, to identify observations consistent with both a priori and emergent themes. ${ }^{30}$ Once consensus among all investigators was achieved, two investigators (EW and JG) independently coded all transcribed notes according to the finalized template. Coders resolved all discrepancies via discussion. All investigators reviewed iterative presentations of final coded data to finalize themes and select prototypic examples.

\section{RESULTS}

Among 50 clinical staff working in the nine clinics, 49 agreed to be observed. Among the 49 participants, 40 (17 RNs, 18 LPNs, and five Health Techs) were observed, and 31 unique staff (15 LPNs, 11 RNs, and five Health Techs) were observed conducting alcohol screening with 72 unique patients (sitespecific numbers reported in Table 1). Qualitative analysis revealed two themes anticipated a priori, three emergent themes specific to conducting alcohol screening with a clinical reminder, and two additional hypothesis-generating themes regarding alcohol screening.

\section{Themes Anticipated a Priori}

Two themes anticipated a priori confirmed known challenges to implementation of care using clinical reminders. ${ }^{9,10,26,27,31-37}$ These included issues related to: 1) workflow, and 2) usability/ flexibility of the reminder. Similar to previous studies, existing workflow often prevented completion of the screening reminder before patient appointments. One nurse participant commented to an observer: "if a provider is trying to stay on time with his/her appointments, they will encourage the nurse to skip the reminders." Another commented, "it's good the patient came early so I have time to do the clinical reminders."

Further, we found that alcohol screening guided by the clinical reminder was inflexible to and did not always optimally address user needs. The clinical reminder often offered clinical staff "no right answers" 26 to click based on the conversations occurring. For instance, patients sometimes offered up recent radical changes in drinking or other important qualitative information, but the clinical reminder did not include a response option or text box to capture this information. In one observation, the Health Tech conducting screening asked "How much do you drink?" and the patient 
Table 1. Number and Type of Observations, by Clinic

\begin{tabular}{|c|c|c|c|c|}
\hline Clinic \# & Clinic Type & Clinical Staff Observed & $\begin{array}{l}\text { Clinical Staff Observed Conducting } \\
\text { Alcohol Screening }\end{array}$ & $\begin{array}{l}\text { Times Alcohol Screening } \\
\text { Observed }\end{array}$ \\
\hline 1 & Contract CBOC* & 1 & 1 & 2 \\
\hline 2 & Contract CBOC* & 1 & 1 & 2 \\
\hline 3 & Contract $\mathrm{CBOC}^{*}$ & 2 & 2 & 8 \\
\hline 4 & VA-Managed CBOC* & 4 & 3 & 4 \\
\hline $5 \mathrm{a}$ & Women's Primary Care Clinic & 3 & 2 & 5 \\
\hline $5 b$ & Large Primary Care Clinic & 11 & 8 & 14 \\
\hline 6 & VA-Managed CBOC* & 5 & 4 & 9 \\
\hline $7 \mathrm{a}$ & Women's Primary Care Clinic & 1 & 1 & 7 \\
\hline & Large Primary Care Clinic & 12 & 9 & 21 \\
\hline TOTAL & & 40 & 31 & 72 \\
\hline
\end{tabular}

*Community-Based Outpatient Clinic, $a$ and $b$ denote different clinics within the same medical center

responded: "I slowed down since I came up here. Maybe two 24 oz cans each day. . .my mother be getting on me about that."

\section{Emergent Themes Regarding Alcohol Screening with a Clinical Reminder}

Three themes specific to conducting alcohol screening with a clinical reminder emerged from the data and reached saturation. Emergent themes and related sub-themes are described in detail below.

\section{Predominantly Verbal Screening Observed Despite Variability in Approaches Across and Within Clinics. Most staff performed alcohol screening verbally by interview, guided by the clinical reminder. However, other methods were observed with variability both within and between clinics. Sometimes a paper form was used (generally only for new patients) that included the AUDIT-C and other behav- ioral health and preventive screenings; it was either mailed or}

self-administered in the waiting room prior to the appointment. When paper-based screening was conducted, the clinical reminder was used to document patients' reported responses. On occasion, staff used laminated paper-based screens to administer the AUDIT-C, whereby clinical staff would hand patients the laminated screen during intake and ask for verbal responses to each question while the staff either input the information directly into the clinical reminder or wrote it down on paper to input later. Regarding this laminate method, one nurse participant commented to an observer: "I think it is more accurate because they can see the answers."

Specific Screening Practices that May Contribute to Low Sensitivity of Alcohol Screening in VA Clinics. We observed four different verbal screening practices, which often occurred in conjunction with one another, that may contribute to low sensitivity of alcohol screening (prototypical examples provided in Table 2). First, the questions were often not asked verbatim. This happened in several different ways. Staff often preceded verbal screening

Table 2. Specific Alcohol Screening Practices that Might Contribute to Low Sensitivity of Clinical Screening

1. Most Verbal Screening Was Not Conducted Verbatim

a. LPN asks patient, "Do you ever drink any type of alcohol? Beer, wine, liquor, mixed drinks?" Patient says, "Yes." Asks, "About how many times per week?" Patient says, "Maybe 3 days." Asks, "About how many drinks at a time?" "Probably 2 or 3." LPN did not ask third question and indicated answer of 0 [never more than 6 per occasion].

b. LPN asks, "Do you drink alcohol?" Patient says," No." LPN clicks "never" for first question and moves on to next reminder

c. "Do you drink alcoholic beverages?" "No". Completes reminder and goes into next reminder.

2. Inferences and/or Assumptions Made Regarding Patient Responses

a. "So how often do you drink, or do you drink?" Patient says, "oh yeah, I do. As a general rule 3 or 4 every day." Health Tech marks Q1 answer 2-3x per week, Q2 answer 5-6 drinks on a typical day, Q3 answer daily or almost daily.

b. Alcohol is the first clinical reminder the LPN opens. She marks less than monthly, 1-2 and never, resulting in a negative screen, but she didn't ever actually ask the patient any alcohol questions

c. "How about in the past year-how often have you had a drink containing alcohol?" Patient answers, "usually 1-2 drinks at a time, or just one." Then patient notes that sometimes that drink is a double. "OK, well we'll just call that 2 then," and clicks on 2 drinks several times a week, though did not clarify frequency with patient.

3. Answers to Alcohol Screening Questions Suggested to Patients

a. Nurse asks, "Any alcohol use in the past year?" Patient: "Oh, yeah." LPN: "What does that mean? Monthly, weekly?" Patient: "Monthly." LPN: "How many?" Patient: "2." LPN: "Never more than 6?" Patient: "Oh, no."

b. "Do you drink alcohol?" "Oh, rarely." "Less than monthly, or more than that?" "Yeah, less than monthly." "Do you usually have 1-2 or what?" "About 1-2" "Never more than 6 at one time?" "Oh no, never more than that."

4. Third Question Regarding Binge Drinking Often Omitted

a. "How often in the last year would you say you had a drink containing alcohol?" Patient looks confused. LPN: "well, like 1 drink a week, one a day, once in a blue moon?" Patient replies, "Not very often. I would say 2 beers a month. I'm on some powerful medications so I can't drink too much." LPN enters: 1-2 drinks for question 1, monthly for question 2, and does not ask third question.

b. LPN asks patient, "How often in the last year did you drink any kind of alcohol?" Patient says, "Maybe 3 times per month." LPN "About 1 or 2 drinks at a time?" Patient says, "Yes." LPN does not ask patient the third question, answers never. 
with questions that were not part of the AUDIT-C (Table 2; examples 1a-1c, 2a, 3a, 3b), and they frequently did not provide patients with the response options (Table 2; examples 1a, 2a, 2c, 3a, 3b, 4a, 4b). Second, staff often made inferences and/or assumptions in selection of AUDIT-C response options when screening was conducted verbally. For instance, some staff documented responses that were not reported into the clinical reminder (Table 2; examples 1a, 2a, 2b), and/or they interpreted general patient responses as fitting into specific AUDIT-C response options (Table 2; examples $2 \mathrm{a}, 4 \mathrm{a}$ ). Third, answers to alcohol screening questions were often suggested by clinical staff based on available response options, before patients had a chance to respond (Table 2; examples 3a, 3b). Fourth, staff often omitted the third question of the AUDIT-C (Table 2; examples 4a, 4b), which asks about frequency of drinking six or more drinks on an occasion.

\section{Staff Introduced and Adapted Screening Questions to} Enhance Patient Comfort. When screening verbally, staff used diverse introductory statements to initiate screening. Some introductions were general, including indicating that the staff in charge of conducting screening was not responsible for deciding who receives screening and clarifying that alcohol screening is routine for all patients. Other introductions appeared specifically focused on enhancing patient comfort (prototypical examples displayed in Table 3). In addition, some staff spontaneously described their perceptions that patients are uncomfortable with alcohol screening and reported adapting the questions in order to make screening more comfortable and acceptable to patients (examples presented in Table 3).

\section{Emergent Themes Without Saturation}

Two additional themes emerged that did not reach saturation, but may affect screening sensitivity. First, staff appeared not to have been trained to conduct alcohol screening in a validated way. Two staff members explicitly expressed that they were not trained. One said, "We all ask the questions in a different way, we have never been taught how to do it." Another said, "Everything is just thrown at us without any training." Second, some staff we observed may have thought screening was targeting identification of patients with the most severe conditions - alcohol use disorders - as opposed to the spectrum of unhealthy alcohol use that also includes risky drinking. ${ }^{38}$ For instance, we observed one interaction in which a nurse commented on his/her feeling that the threshold for positive screening was low: "The VA is very tough on alcohol... if you don't drink much, they say 'don't drink too much.',

\section{DISCUSSION}

Observations of clinical staff conducting alcohol screening at nine independently-managed primary care clinics showed that staff most often conducted alcohol screening verbally. Verbal screening included practices that may result in underidentification of unhealthy alcohol use, including asking the questions non-verbatim; making inferences, assumptions, and/ or suggestions of responses to the questions; omitting the third AUDIT-C question regarding frequency of binge drinking; and otherwise adapting the questions. Previous studies have found high rates of documented screening with VA's electronic clinical reminder, ${ }^{9,20}$ but found that clinical screening missed many patients with unhealthy alcohol use. ${ }^{20}$ Findings from the present qualitative study regarding specific verbal screening practices help explain those findings.

Findings from this study also suggest several possible reasons that screening questions were modified when screening was conducted verbally. Specifically, consistent with previous research, ${ }^{26,27}$ this study identified issues with existing workflow, such that completion of the alcohol screening clinical reminder prior to patients' appointments was sometimes impractical. Though it is unknown why clinical staff modify questions, they may be attempting to make them briefer in order to fit into existing workflow. In addition, this study's

Table 3 Examples of Introductory Statements and Reports of Adaptating Screening to Enhance Patient Comfort

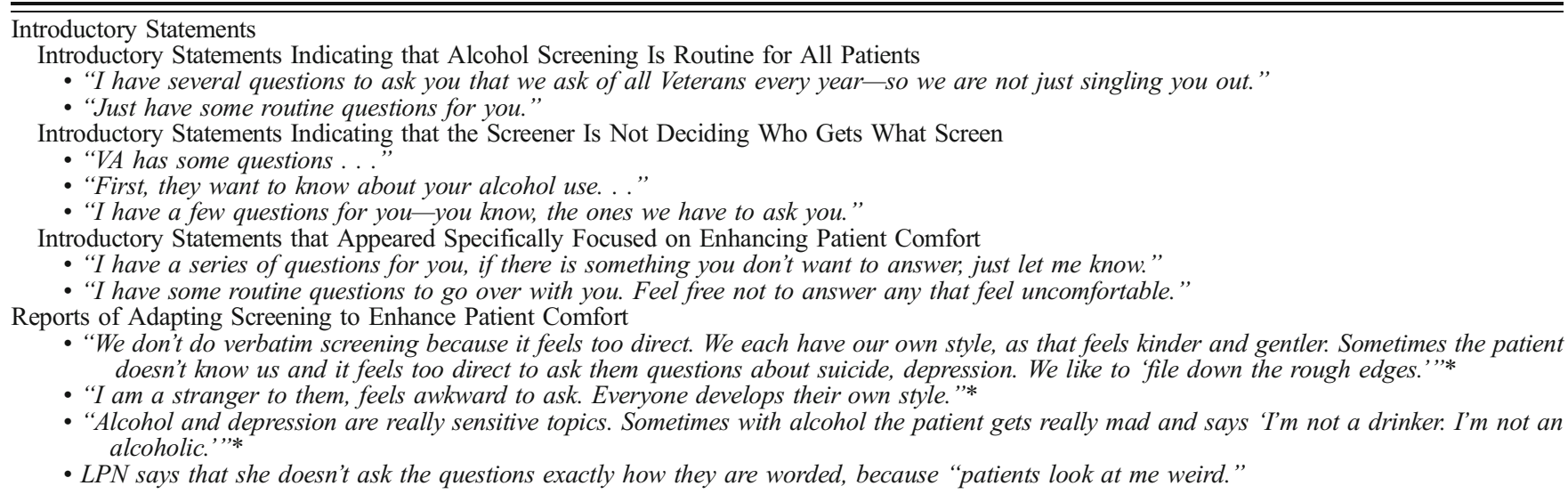


findings suggested that staff introduced and adapted questions in order to address their perceptions that patients were not comfortable with alcohol screening. It is unknown whether these practices reflected actual patient discomfort, staff's own discomfort, and/or general social stigma related to alcohol use. ${ }^{39}$ Regardless of their underlying cause, and the fact that these efforts are likely aimed at making patients more comfortable and care more patient-centered, resulting adaptations to screening questions may diminish the sensitivity of screening.

Findings from this study may have implications for other healthcare systems implementing alcohol screening, as well as screening for other important substance use and mental health conditions. ${ }^{21-24,40}$ Although not without limitation, ${ }^{41,42}$ clinical decision support systems have been used to implement evidence-based practices across multiple conditions, ${ }^{43-49}$ and many of these systems are being developed to be shareable across multiple healthcare systems, ${ }^{50}$ especially in the U.S. in response to healthcare reform. Findings from the present and prior studies in VA suggest that while VA's alcohol screening clinical reminder has effectively prompted clinical staff to conduct screening and facilitated documentation that screening occurred, ${ }^{9}$ it has not facilitated screening in a validated, standardized, reproducible way.

If healthcare systems use clinical reminders to implement screening for unhealthy alcohol use and other mental health conditions, additional implementation strategies may be needed to optimize quality (i.e., to successfully identify the patients who might benefit from indicated interventions). Specifically, healthcare systems may need to specify a preferred approach to screening. If verbal screening is recommended, successful implementation $^{30,51}$ may rely on strategies that actively engage clinicians. ${ }^{48,52-54}$ Our hypothesis-generating findings suggested clinical staff may not have been systematically trained to conduct alcohol screening, and may be not be aware that screening should identify patients with risky drinking in addition to those with alcohol use disorders. ${ }^{55-57}$ User-level training strategies, such as didactic training, academic detailing, clinical champions, or practice facilitation ${ }^{58,59}$ may be necessary in order to convey risks associated with the entire spectrum of unhealthy alcohol use (including risky drinking), and the reason for identifying patients with the spectrum of unhealthy alcohol use (i.e., the efficacy of brief intervention).

However, while application of additional strategies, including user-level training, may contribute to a clinical culture with greater aptitude for offering high-quality alcohol-related care, these strategies may be resourceheavy and cost prohibitive. Further, findings from the present study call into question whether brief mental health screens, and particularly the AUDIT-C or other screens with multiple specified response options, should be administered verbally by a clinical interviewer, or if they should instead be patient administered. Patient selfadministered screening, such as laminate, paper-based, or web-based screening, may address underlying reasons for non-standard screening (i.e., workflow issues and responses to perceptions of patient discomfort), avoid the need for continual training of new staff, and increase the sensitivity of clinical screening above that achieved with interviewer administered screening. ${ }^{60}$

This study has several limitations. First, practices observed at the study clinics may not be generalizable to those at other VA healthcare systems (of which there are approximately 150 nationally). Future research is needed to assess whether results are similar in other VA and nonVA healthcare settings/clinics. Generalizablity may also have been limited by requiring agreement to be observed from clinical staff participants and their patients. While all but one staff agreed to be observed, not all who agreed were observed due to limited research staff and time in clinics, and not all who were observed conducted alcohol screening because, by design, patients were not considered subjects of the research, and thus we were unable to determine a priori which patients were "due" for alcohol screening. Second, observations may not be entirely objective or reflective of existing clinical care-research staff did not audio-record or video-record clinical screening and were not blinded to the study purpose, which may have influenced their notes. In addition, staff were aware they were being observed, which may have altered their practices. Third, although our observations suggested reasons underlying the low sensitivity of clinical alcohol screening in VA, this study did not specifically test whether and how staff delivery of questions impacts patient responses or screening validity. Finally, although some staff offered us their opinions or experience of screening, we did not systematically elicit their experiences. Therefore, future research is needed to understand perspectives and experiences of clinical staff who conduct screening.

Despite these limitations, this large qualitative study found that implementation of alcohol screening facilitated by a clinical reminder resulted primarily in verbal screening, which was often not conducted in a standardized, validated fashion. Issues related to workflow, efforts to make patients comfortable, and lack of training may have resulted in observed screening practices. As healthcare systems move forward with implementation of alcohol screening, as well as screening for other mental health and substance use conditions, use of a clinical reminder alone may be limited as a method of facilitating valid, standardized screening. Systems may need to specify a preferred approach to screening. Patient self-administration of recommended mental health and substance use screens (e.g., laminate, paper-based, or web-based patient self-screening) may address 
underlying reasons for adapting screening questions, and thus, offer a strong alternative to verbal approaches to screening.

Acknowledgements: The authors gratefully acknowledge the participants of this study, as well as their patients, for generously allowing us to observe them. We also wish to acknowledge Jason Saleem, $\mathrm{PhD}$, for inspiration, as well as for sharing his experience and advice regarding qualitative studies of clinical reminder use in the VA during study design.

This study was funded by VA Health Services Research \& Development and VA Quality Enhancement Research Initiative (RRP 09-178). Dr. Williams is supported by a Career Development Award from VA Health Services Research \& Development (CDA 12-276), and is an investigator with the Implementation Research Institute (IRI) at the George Warren Brown School of Social Work at Washington University in St. Louis. IRI is supported through an award from the National Institute of Mental Health (R25 MH080916-01A2) and the Department of Veterans Affairs, Health Services Research \& Development Service, Quality Enhancement Research Initiative (QUERI). Dr. Bradley's time on this study was supported by the Center of Excellence for Substance Abuse Treatment and Education (CESATE) at VA Puget Sound. Ms. Chavez is supported by an Agency for Healthcare Research and Quality (AHRQ) Dissertation Grant (NIH 1R36HSO22800-01).

Preliminary findings were presented at two previous annual meetings of the International Network on Brief Interventions for Alcohol \& Other Drugs (Boston, MA, September 2011 and Rome, Italy, September 2013) and at the annual meeting of the VA Health Services Research \& Development service (Washington DC, February 2012).

Conflict of Interest Statement: Dr. Bradley owns stocks in four pharmaceutical companies: Abbvie, Johnson and Johnson, Pfizer, and Proctor and Gamble. All other authors declare that they do not have a conflict of interest.

Corresponding Author: Emily C. Williams, $P h D, M P H$; VA Puget Sound Health Care System, 1100 Olive Way, Suite 1400, Seattle, WA 98101, USA (e-mail: emily.williams3@va.gov).

\section{REFERENCES}

1. National Institute on Alcohol Abuse and Alcoholism. Helping Patients Who Drink Too Much: A Clinician's Guide (Updated 2005 Edition). Washington: National Institutes of Health, U.S. Department of Health and Human Services; 2007

2. Jonas DE, Garbutt JC, Amick HR, et al. Behavioral counseling after screening for alcohol misuse in primary care: a systematic review and meta-analysis for the U.S. Preventive Services Task Force. Ann Intern Med. 2012;157(9):645-54.

3. National Health Service. National Institute for Health and Clinical Excellence: Alcohol-use disorders: preventing harmful drinking. NICE public health guidance 24; http://www.nice.org.uk/guidance/CG115.

4. Maciosek MV, Coffield AB, Edwards NM, Flottemesch TJ, Goodman MJ, Solberg LI. Priorities among effective clinical preventive services results of a systematic review and analysis. Am J Prev Med 2006;31(1):52-61. see erratum page 458 Volume 432, Number 455.

5. Solberg LI, Maciosek MV, Edwards NM. Primary care intervention to reduce alcohol misuse ranking its health impact and cost effectiveness. Am J Prev Med. 2008;34(2):143-52.

6. Williams EC, Johnson ML, Lapham GT, et al. Strategies to implement alcohol screening and brief intervention in primary care settings: a structured literature review. Psychol Addict Behav. 2011;25(2):206-14.

7. Nilsen P. Brief alcohol intervention-where to from here? Challenges remain for research and practice. Addiction. 2010;105(6):954-9.

8. Nilsen $\mathbf{P}$, Aalto $\mathbf{M}$, Bendtsen $\mathbf{P}$, Seppa $\mathbf{K}$. Effectiveness of strategies to implement brief alcohol intervention in primary healthcare. A systematic review. Scand J Prim Health Care. 2006;24(1):5-15.

9. Bradley KA, Williams EC, Achtmeyer CE, Volpp B, Collins BJ, Kivlahan DR. Implementation of evidence-based alcohol screening in the Veterans Health Administration. Am J Manage Care. 2006;12(10):597-606.
10. Bradley KA, Williams EC. Implementation of Screening and Brief Intervention in Clinical Settings Using Quality Improvement Principles. In: Fiellin D, Miller S, Saitz R, Reis R, eds. Principles of Addiction Medicine 4th ed: Lippincott Williams \& Wilkins; 2009:288-92.

11. Moyer A, Finney JW. Meeting the challenges for research and practice for brief alcohol intervention. Addiction. 2010;105(6):963-4. discussion 964-965.

12. Kerr EA, Fleming B. Making performance indicators work: experiences of US Veterans Health Administration. BMJ. 2007;335(7627):971-3.

13. Kizer KW, Dudley RA. Extreme makeover: Transformation of the Veterans Health Care System. Annu Rev Public Health. 2009;30:313-39.

14. Bradley KA, Bush KR, Epler AJ, et al. Two brief alcohol-screening tests from the Alcohol Use Disorders Identification Test (AUDIT): validation in a female Veterans Affairs patient population. Arch Intern Med. 2003; 163(7):821-9.

15. Dawson DA, Grant BF, Stinson FS, Zhou Y. Effectiveness of the derived Alcohol Use Disorders Identification Test (AUDIT-C) in screening for alcohol use disorders and risk drinking in the US general population. Alcohol Clin Exp Res. 2005;29(5):844-54.

16. Bush K, Kivlahan DR, McDonell MB, Fihn SD, Bradley KA. The AUDIT alcohol consumption questions (AUDIT-C): an effective brief screening test for problem drinking. Ambulatory Care Quality Improvement Project (ACQUIP). Alcohol Use Disorders Identification Test. Arch Intern Med. 1998; 158(16):1789-95.

17. Frank D, DeBenedetti AF, Volk RJ, Williams EC, Kivlahan DR, Bradley KA. Effectiveness of the AUDIT-C as a screening test for alcohol misuse in three race/ethnic groups. J Gen Intern Med. 2008;23(6):781-7.

18. Bradley KA, DeBenedetti AF, Volk RJ, Williams EC, Frank D, Kivlahan DR. AUDIT-C as a brief screen for alcohol misuse in primary care. Alcohol Clin Exp Res. 2007;31(7):1208-17.

19. Hawkins EJ, Kivlahan DR, Williams EC, Wright SM, Craig T, Bradley KA. Examining quality issues in alcohol misuse screening. Subst Abus. 2007;28(3):53-65.

20. Bradley KA, Lapham GT, Hawkins EJ, et al. Quality concerns with routine alcohol screening in VA clinical settings. J Gen Intern Med. 2011;26(3):299-306.

21. Centers for Medicare and Medicaid Services. Decision Memo for Screening and Behavioral Counseling Interventions in Primary Care to Reduce Alcohol Misuse (CAG-00427N) Available from: http://www.cms.gov/medicare-coverage-database/details/nca-decision-memo.aspx?NCAId=249\#_ ftn29. 2011. Accessed February 9, 2015.

22. HealthCare.gov. What does marketplace health insurance cover? https:// www.healthcare.gov/what-does-marketplace-health-insurance-cover/. 2013. Accessed February 9, 2015.

23. HealthCare.gov. What are my preventive care benefits? https://www. healthcare.gov/what-are-my-preventive-care-benefits/. 2013. Accessed February 9, 2015.

24. Mertens J, Sterling S, Weisner C, Brumder-Ross T. Alcohol SBIRT Implementation in Adult Primary Care: Physician versus Non-Physician Delivery. Jennifer Mertens, S Sterling, C Weisner,T Brumder-Ross. Kaiser Permanente Division of Research, Oakland, USA Paper presented at: International Network on Brief Interventions for Alcohol \& Other Drugs (INEBRIA) 2013; Rome, Italy.

25. Williams EC, Rubinsky AD, Chavez LJ, et al. An early evaluation of implementation of brief intervention for unhealthy alcohol use in the US Veterans Health Administration. Addiction. 2014;109(9):1472-81.

26. Saleem JJ, Patterson ES, Militello L, Render ML, Orshansky G, Asch SM. Exploring barriers and facilitators to the use of computerized clinical reminders. J Am Med Inform Assoc. 2005;12(4):438-47.

27. Patterson ES, Nguyen AD, Halloran JP, Asch SM. Human factors barriers to the effective use of ten HIV clinical reminders. J Am Med Inform Assoc. 2004; 11(1):50-9.

28. King N. Template Analysis. In: Symon G, Cassell C, and Hallam S, eds. Qualitative Methods and Analysis in Organizational Research. London: Sage Publications; 1998:118-34.

29. Bradley EH, Curry LA, Devers KJ. Qualitative data analysis for health services research: developing taxonomy, themes, and theory. Health Serv Res. 2007:42(4): 1758-72.

30. Greenhalgh T, Robert G, Macfarlane F, Bate P, Kyriakidou O. Diffusion of innovations in service organizations: systematic review and recommendations. Milbank Q. 2004;82(4):581-629.

31. Fung $\mathbf{C H}$, Tsai JS, Lulejian A, et al. An evaluation of the Veterans Health Administration's clinical reminders system: a national survey of generalists. J Gen Intern Med. 2008;23(4):392-8.

32. Fung CH, Woods JN, Asch SM, Glassman P, Doebbeling BN. Variation in implementation and use of computerized clinical reminders in an integrated healthcare system. Am J Manage Care. 2004;10(11 Pt 2):878-85. 
33. Militello L, Patterson ES, Tripp-Reimer T, et al. Clinical reminders: why don't they use them? Paper presented at: Human Factors and Ergonimics Society 48th Annual Meeting 2004.

34. Patterson ES, Doebbeling BN, Fung CH, Militello L, Anders S, Asch SM. Identifying barriers to the effective use of clinical reminders: bootstrapping multiple methods. J Biomed Inform. 2005;38(3):189-99.

35. Mayo-Smith MF, Agrawal A. Factors associated with improved completion of computerized clinical reminders across a large healthcare system. Int $\mathrm{J}$ Med Inform. 2007;76(10):710-6.

36. Krall MA, Sitting DF. Clinicians' assessments of outpatient electronic medical record alert and reminder usability and usefulness requirements. Proc Amia Symp. 2002:400-404.

37. Bates DW, Kuperman GJ, Wang S, et al. Ten commandments for effective clinical decision support: making the practice of evidence-based medicine a reality. J Am Med Inform Assoc. 2003;10(6):523-30.

38. Saitz R. Clinical practice. Unhealthy alcohol use. N Engl J Med. 2005;352(6):596-607.

39. Link BG, Struening EL, Rahav M, Phelan JC, Nuttbrock L. On stigma and its consequences: evidence from a longitudinal study of men with dual diagnoses of mental illness and substance abuse. J Health Soc Behav. 1997;38(2):177-90.

40. Keurhorst MN, Anderson P, Spak F, et al. Implementing training and support, financial reimbursement, and referral to an internet-based brief advice program to improve the early identification of hazardous and harmful alcohol consumption in primary care (ODHIN): study protocol for a cluster randomized factorial trial. Implement Sci. 2013;8:11.

41. Powell AA, White KM, Partin MR, et al. Unintended consequences of implementing a national performance measurement system into local practice. J Gen Intern Med. 2012;27(4):405-12.

42. Kansagara D, Tuepker A, Joos S, Nicolaidis C, Skaperdas E, Hickam D. Getting Performance Metrics Right: A Qualitative Study of Staff Experiences Implementing and Measuring Practice Transformation. J Gen Intern Med. Jul 2014; Suppl 2:S607-613.

43. Hunt DK, Haynes BR, Hanna SE, Smith K. Effects of computer-based clinical decision support systems on physician performance and patient outcomes: A systematic review. JAMA. 1998;280(15):1339-46.

44. Shea S, DuMouchel W, Bahamonde L. A meta-analysis of 16 randomized controlled trials to evaluate computer-based clinical reminder systems for preventive care in the ambulatory setting. J Am Med Inform Assoc. 1996;3(6):399-409.

45. Kitahata MM, Dillingham PW, Chaiyakunapruk N, et al. Electronic human immunodeficiency virus (HIV) clinical reminder system improves adherence to practice guidelines among the University of Washington HIV Study Cohort. Clin Infect Dis. 2003;36(6):803-11.
46. Goetz MB, Hoang T, Knapp H, et al. Central Implementation Strategies Outperform Local Ones in Improving HIV Testing in Veterans Healthcare Administration Facilities. J Gen Intern Med. 2013;28(10):1311-7.

47. Kralj B, Iverson D, Hotz K, Ashbury FD. The impact of computerized clinical reminders on physician prescribing behavior: evidence from community oncology practice. Am J Med Qual. 2003;18(5):197-203.

48. Lainer M, Mann E, Sonnichsen A. Information technology interventions to improve medication safety in primary care: a systematic review. Int $\mathrm{J}$ Qual Health Care. 2013;25(5):590-8.

49. Montini T, Schenkel AB, Shelley DR. Feasibility of a computerized clinical decision support system for treating tobacco use in dental clinics. J Dent Educ. 2013;77(4):458-62.

50. Goldberg HS, Paterno MD, Rocha BH, et al. A highly scalable, interoperable clinical decision support service. J Am Med Inform Assoc. 2013;21(e1):e55-62.

51. Damschroder LJ, Aron DC, Keith RE, Kirsh SR, Alexander JA, Lowery JC. Fostering implementation of health services research findings into practice: a consolidated framework for advancing implementation science. Implement Sci. 2009;4:50.

52. Prior M, Guerin M, Grimmer-Somers K. The effectiveness of clinical guideline implementation strategies-a synthesis of systematic review findings. J Eval Clin Pract. 2008; 14(5):888-97.

53. Kealey E, Leckman-Westin E, Finnerty MT. Impact of four training conditions on physician use of a web-based clinical decision support system. Artif Intell Med. 2013;59(1):39-44.

54. Millery M, Shelley D, Wu D, Ferrari P, Tseng TY, Kopal H. Qualitative evaluation to explain success of multifaceted technology-driven hypertension intervention. Am J Manage Care. 2011;17(12 Spec No):SP95-102.

55. Saitz R, Fiellin DA. Do brief interventions for unhealthy alcohol use work? Intern Med News. 2005.

56. Saitz R, Svikis D, D'Onofrio G, Kraemer KL, Perl H. Challenges applying alcohol brief intervention in diverse practice settings: populations, outcomes, and costs. Alcohol Clin Exp Res. 2006;30(2):332-8.

57. Guth S, Lindberg SA, Badger GJ, Thomas CS, Rose GL, Helzer JE. Brief intervention in alcohol-dependent versus nondependent individuals. J Stud Alcohol Drugs. 2008;69(2):243-50.

58. Ludman EJ, Curry SJ, Grothaus LC. A longitudinal assessment of physician attitudes, beliefs and practice following academic detailing and chart cues for prevention of alcohol problems. Alcohol Res. 2004;9(1):12-8.

59. Baskerville NB, Liddy C, Hogg W. Systematic review and meta-analysis of practice facilitation within primary care settings. Ann Fam Med. 2012;10(1):63-74.

60. Wu RR, Orlando LA, Himmel TL, et al. Patient and primary care provider experience using a family health history collection, risk stratification, and clinical decision support tool: a type 2 hybrid controlled implementationeffectiveness trial. BMC Fam Pract. 2013;14(1):111. 